\title{
Monte Carlo simulation of light-emitting diode light-extraction characteristics
}

\author{
David Z.-Y. Ting \\ Thomas C. McGill \\ California Institute of Technology \\ Thomas J. Watson, Sr., Laboratory of \\ Applied Physics \\ Pasadena, California 91125
}

\begin{abstract}
We employ a Monte Carlo ray-tracing technique to model lightextraction characteristics of light-emitting diodes. By keeping track of the various processes that photons can encounter in a packaged LED, consisting of a semiconductor chip, a reflector dish, and an epoxy magnifying lens, our model can provide valuable information for LED design. The simulations can be performed very effectively on modern workstations. Preliminary results from a sample parametric study indicate that, with some refinement, our method could be used as a computer-aided design tool for optimizing light-extraction efficiencies and for designing LED spatial emission characteristics.
\end{abstract}

Subject terms: LED modeling; light-extraction, Monte Carlo.

Optical Engineering 34(12), 3545-3553 (December 1995).

\section{Introduction}

In the past 25 years, the luminous performance of LEDs has improved $^{1}$ by a factor of 100. Today's high-performance LEDs are challenging incandescent light sources in brightness and efficiency. ${ }^{2}$ The external quantum efficiency of an LED is the product of its internal quantum efficiency, which measures how effectively injected carriers are converted into photons, and optical (extraction) efficiency, which indicates how easily photons can be extracted from the semiconductor LED chip. Both are equally important in determining LED performance. Extraction efficiency is usually substantially less than 1. Photon extraction from LED chips is difficult for several reasons. Absorption by the medium and contacts prevent many of the internally generated photons from ever reaching exposed chip surfaces in the first place. A substantial fraction of the photons that do reach the surface end up being internally reflected because critical angles at chip surfaces are typically small because of the large index of refraction mismatch between the semiconductor LED chip and its surrounding medium. For example, for a chip with index of refraction of 3.1 , the critical angle at a chip-air interface is only $19 \mathrm{deg}$. The angle can be increased if the chip is encapsulated in an epoxy with an intermediate index. For an epoxy with an index of 1.7, the critical angle is $33 \mathrm{deg}$, which is still fairly small.

Two analytical models developed in the past are conceptually very useful for considering light extraction from LED chips in limiting cases. Carr ${ }^{3}$ considered the situation where

Paper 34025 received Feb. 27, 1995; revised manuscript received May 13, 1995; accepted for publication May 15, 1995.

(C) 1995 Society of Photo-Optical Instrumentation Engineers. 0091-3286/95/\$6.00. the absorption length is small compared to overall semiconductor chip dimensions. In that case, most of the internally reflected rays would be absorbed, and only photons striking chip surfaces at below the critical angle need to be accounted for. Joyce et al. ${ }^{4}$ considered the case of weak absorption, where the photon flux inside the semiconductor chip is highly randomized by multiple internal reflections and rough surfaces, leading to Lambertian emission patterns from chip surfaces. We approach the problem of modeling LED light extraction with a numerical Monte Carlo ray-tracing technique, which has a greater range of validity than the limiting cases treated by the analytical models. Our method is made feasible by the ready availability of high-speed computation platforms. On a modern workstation, it is possible to perform a simulation using 2 million photons in under $1 \mathrm{~min}$, even with extensive statistics collection. In this paper, we demonstrate that, with some refinement, our method could be used in a computer-aided design tool for customizing emission patterns and optimizing light-extraction efficiencies in LEDs. The remainder of this paper is organized as follows: we describe our method in Sec. 2, present results on an illustrative example in Sec. 3, and summarize in Sec. 4.

\section{Model}

We first specify the LED lamp geometry used in our model, and then describe the Monte Carlo simulation procedure.

\subsection{Geometry}

There are a number of industry standard LED lamp geometries in use today. ${ }^{5}$ In our calculations, we used the T-13/4 LED lamp, illustrated in Fig. 1, as our prototype structure. In this structure, the light-emitting semiconductor chip is set in a reflector dish coined into the cathode post of the lead 


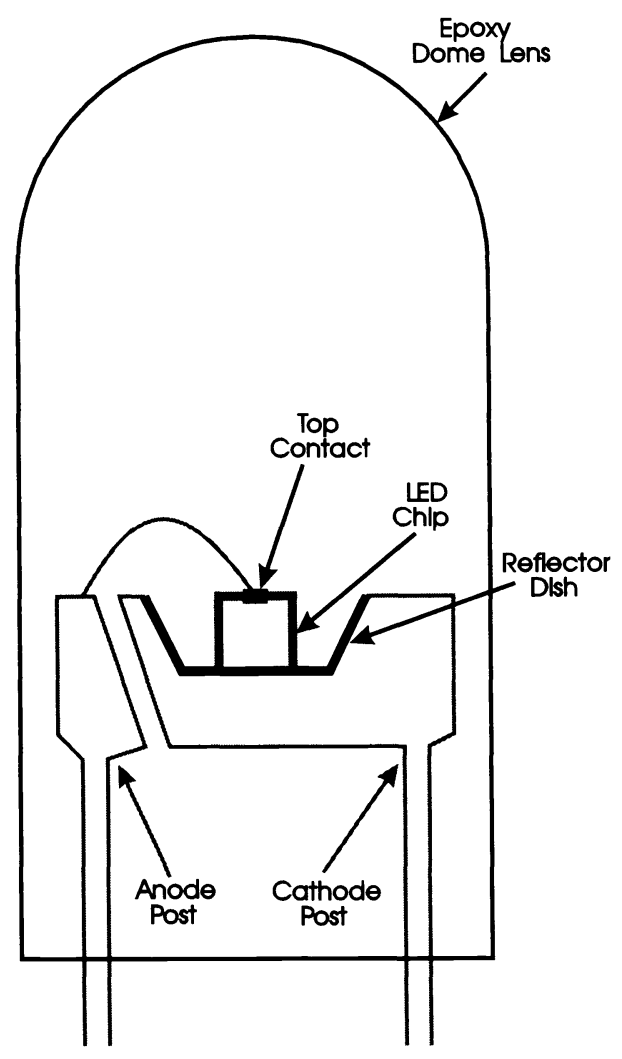

Fig. 1 Illustration of the T-13/4 LED lamp (not to scale) used in our simulations. Note that the anode post, cathode post, and the bonding wire are ignored in our simulations.

frame. The top contact on the chip is wire bonded to the anode post, and the entire lead-frame/chip assembly is encapsulated in an epoxy magnifying dome lens. In our simulations, we ignore the lead frame and the bonding wire, and concentrate on the four components that are most important for modeling light-extraction characteristics, namely, the LED chip, light-absorbing contacts, the reflector dish, and the epoxy lens.

We choose the principal axis of the epoxy lens to be the $z$ axis of our coordinate system, with $z=0$ set at the bottom of the LED chip. The LED chip is a rectangular solid of dimensions $w, d$, and $h$, with a top circular contact of radius $r_{c}$. The light-emitting $p$ - $n$ junction in the chip is located at $z=h_{\text {junct }}$. The reflector dish consists of a circular disk of radius $r_{d}$, and a sidewall described by the equation of a truncated cone, given by

$x^{2}+y^{2}=\left[\gamma\left(z-z_{0}\right)\right]^{2}, \quad z \in\left[0, h_{d}\right]$.

Here $\gamma$ is related to the slope of the sidewall, $z_{0}$ locates the tip of the cone, and $h_{d}$ is the height of the dish sidewall. Note that $r_{d}=\left|\gamma z_{0}\right|$. The epoxy lens consists of a hemispheric dome set on top of a cylinder. The hemisphere has a radius of $r_{l}$, and is centered at $z=z_{l}$ on the $z$ axis. The cylinder extends from $z=z_{l B}$ to $z=z_{l T}$, and has a radius of $r_{l}$. We assume the semiconductor chip is homogeneous, or more precisely, we assume that medium absorption properties can be described by a single absorption coefficient spectrum $\alpha(E)$. Of course, our model can be modified to accommodate heterostructures.
We used $n_{c}, n_{l}$, and $n_{0}$ to denote the indices of refraction of the chip, lens, and air, respectively.

\subsection{Simulation Procedure}

We use a Monte Carlo particle simulation approach to model LED light-extraction characteristics. The particles used are classical photons (corpuscles), which obey the rules of geometrical optics. A simulation is performed with an ensemble of randomly generated photons, and the processes that each photon encounters (e.g., medium absorption, transmission at interfaces) are governed by probabilistic models.

We assume that photons are generated uniformly within a thin planar region at $z=h_{\text {junct }}$, and are emitted in an isotropic spatial pattern. ${ }^{3}$ We also assume monochromatic photon emission for simplicity. A trivial extension to our model would allow for the treatment of more general emission spectra. For each photon, a position vector on the emission plane and a direction vector are randomly generated. A ray-polygon intersection algorithm ${ }^{6}$ is used to compute the intersection of photon trajectory with one of the six chip surfaces. Because a photon might be absorbed inside the chip before reaching the destination surface, we use a Monte Carlo routine to determine whether absorption takes place. Let the path length between the initial and final positions be $\Delta$. The probability of a photon being absorbed in a distance $\Delta$ is given by

$P(\Delta)=1-e^{-\alpha \Delta}$,

where $\alpha$ is the medium absorption coefficient. We generate a random number $R$ between 0 and 1; absorption takes place if $R \leqslant P(\Delta)$. If a photon reaches a destination chip surface without being absorbed by the semiconductor, we check to see if it strikes either the top or the bottom contact, where contact absorption processes can occur. For simplicity, we assume that the contacts are totally absorbing, although partially absorbing contacts can be modeled quite easily. If the photon is not absorbing, we determine whether it is transmitted or reflected by generating a random number between 0 and 1 , and comparing it to the transmission probability given by the Fresnel equations (see, e.g., Ref. 7). An internally reflected photon continues on its path within the LED chip until it is eventually absorbed or transmitted.

A photon emitted by the LED chip may undergo one of the processes illustrated in Fig. 2. These processes include direct escape without striking the reflector dish, reflector dish sidewall reflection, disk reflection, and combination disk-wall

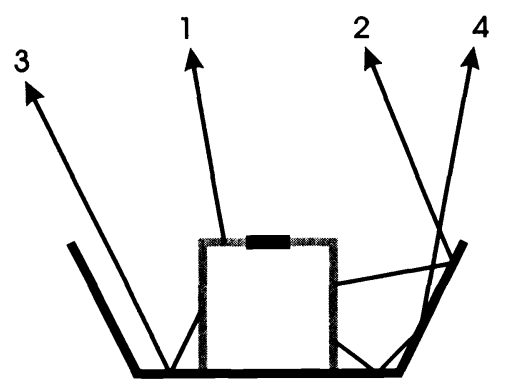

Fig. 2 Illustration of reflector dish processes: (1) direct escape without hitting dish, (2) cone reflection, (3) disk reflection, and (4) diskcone combination. 
Table 1 LED structure parameters used in this calculation.

\begin{tabular}{|c|c|c|c|c|c|}
\hline \multicolumn{2}{|c|}{ Semiconductor Chip } & \multicolumn{2}{|c|}{ Reflector Dish } & \multicolumn{2}{|c|}{ Epoxy Lens } \\
\hline$w(\mu \mathrm{m})$ & 266.7 & $\gamma$ & 1.0 & $r_{l}(\mathrm{~cm})$ & 0.250 \\
\hline$d(\mu \mathrm{m})$ & 266.7 & $z_{0}(\mu \mathrm{m})$ & -254.0 & $z_{l T}(\mathrm{~cm})$ & 0.246 \\
\hline$h(\mu \mathrm{m})$ & 266.7 & $h_{d}(\mu \mathrm{m})$ & 304.8 & $z_{l B}(\mathrm{~cm})$ & -0.380 \\
\hline$r_{c}(\mu \mathrm{m})$ & 66.0 & & & & \\
\hline
\end{tabular}

reflection. In this work, we do not take into account the possibility of photon re-entrance into the LED chip; we address the implications of this issue at the end of the next section. A photon emanating from the chip-reflector dish assembly bounces around inside the lens until it escapes through the dome, the cylinder wall, or the bottom. As before, the Fresnel equations are used to determine whether reflection or transmission occurs at lens-air interfaces. Also, re-entrance into the chip-dish assembly is not considered. Throughout the different stages of the simulation, extensive statistics are gathered for subsequent analyses.

\section{Results and Discussion}

We apply our method to a T-13/4 LED lamp; the structure parameters for this device are listed in Table 1 . We assume that the index of refraction for the semiconductor chip and the epoxy are $n_{c}=3.1$ and $n_{l}=1.7$, respectively, ${ }^{*}$ and that the absorption coefficient for the semiconductor chip is $\alpha=10 \mathrm{~cm}^{-1}$. This corresponds to a weakly absorbing medium, with a characteristic absorption length $1 / \alpha$ of $1000 \mu \mathrm{m}$, or 3.75 times the width of the cubic chip. Admittedly, our choice for the value of $\alpha$ is somewhat artificial; we chose this value so that various chip processes would occur in sufficient numbers to be of interest in our demonstration. A simulation of a realistic device would require that we use experimentally measured absorption coefficient spectrum $\alpha(E)$ for the LED material.

In this paper, we demonstrate the potential usefulness of our Monte Carlo simulation method. We illustrate this with a hypothetical case study where we vary $h_{\text {junct }}$ to examine the dependence of light-emission characteristics on the height of the light-emitting junction plane.

Figure 3 shows the light-extraction efficiency for the LED as a function of the light-emitting junction height, which ranges from 0 to $265 \mu \mathrm{m}$ (the height of the chip is $266.7 \mu \mathrm{m}$, or 1.05 mil.). The efficiency is low when the junction is near the bottom of the chip. As the junction is raised, the efficiency increases until reaching a maximum at $h_{\text {junct }}=190 \mu \mathrm{m}$, and then decreases. At its maximum, the efficiency is nearly twice the $h_{\text {junct }}=0$ value. We can understand this trend by examining the processes occurring in the semiconductor chip in more detail. Figure 4 shows the fractions of photons undergo-

\footnotetext{
*We chose an index of refraction for the epoxy to be close to the geometric mean between chip and air indices. However, it has been pointed out to us that, in practice, epoxy indices do not vary much from the value around 1.5 , and do not exceed 1.51. This should not change the qualitative results presented in this paper.
}

ing various processes in the semiconductor chip as functions of $h_{\text {junct }}$. For small values of $h_{\text {junct }}$, bottom contact absorption is the dominant process, accounting for more than half of the photons. This is because we have assumed that the contacts are totally absorbing. Therefore, for small $h_{\text {junct }}$, all downward-pointing photons generated at the junction (half of all photons generated) are immediately absorbed by the bottom contact. As the junction height increases, the fraction of photons absorbed by the bottom contact decreases considerably. The fraction of photons emitted through the top surface is comparable to that for photons absorbed by the top contact, even though the top contact only covers approximately $19 \%$ of the top surface. This is because only those photons reaching the exposed portion of the top surface at an angle less than the critical angle (33 deg in this case) could escape. Both medium absorption and side emission fractions remain fairly constant as functions of $h_{\text {junct }}$, except for low values of junc-

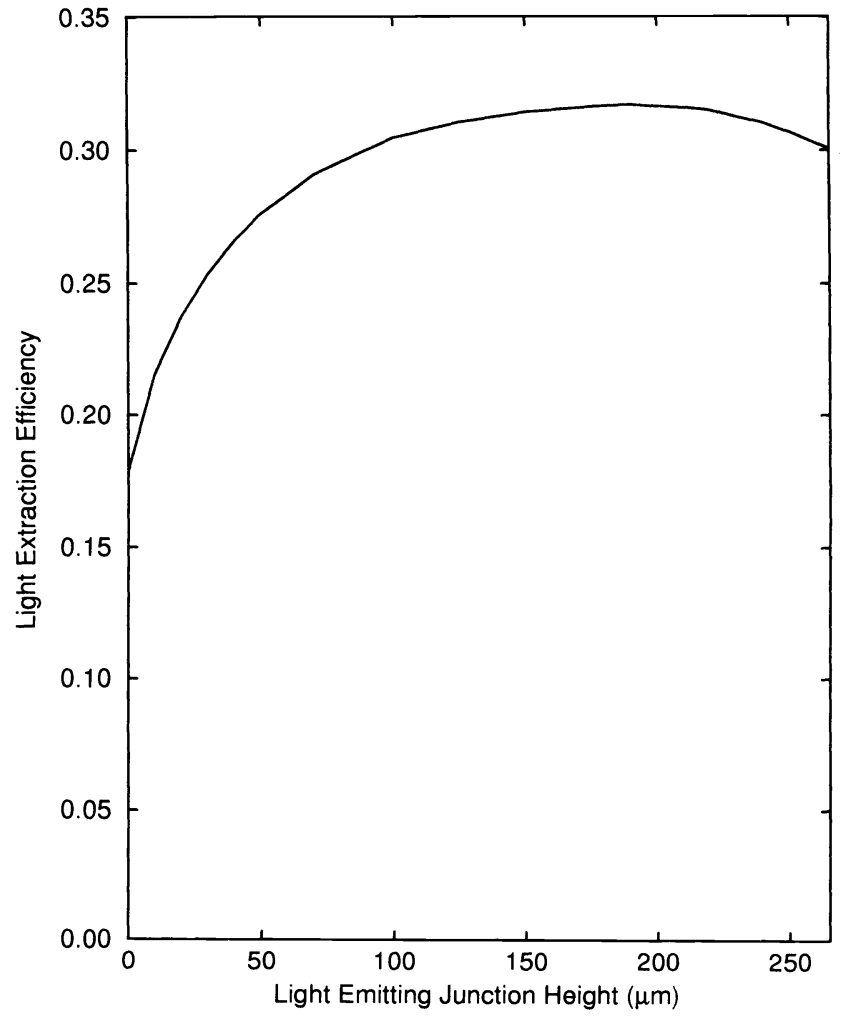

Fig. 3 Computed photon extraction efficiency as a function of lightemitting junction height in LED chip. 


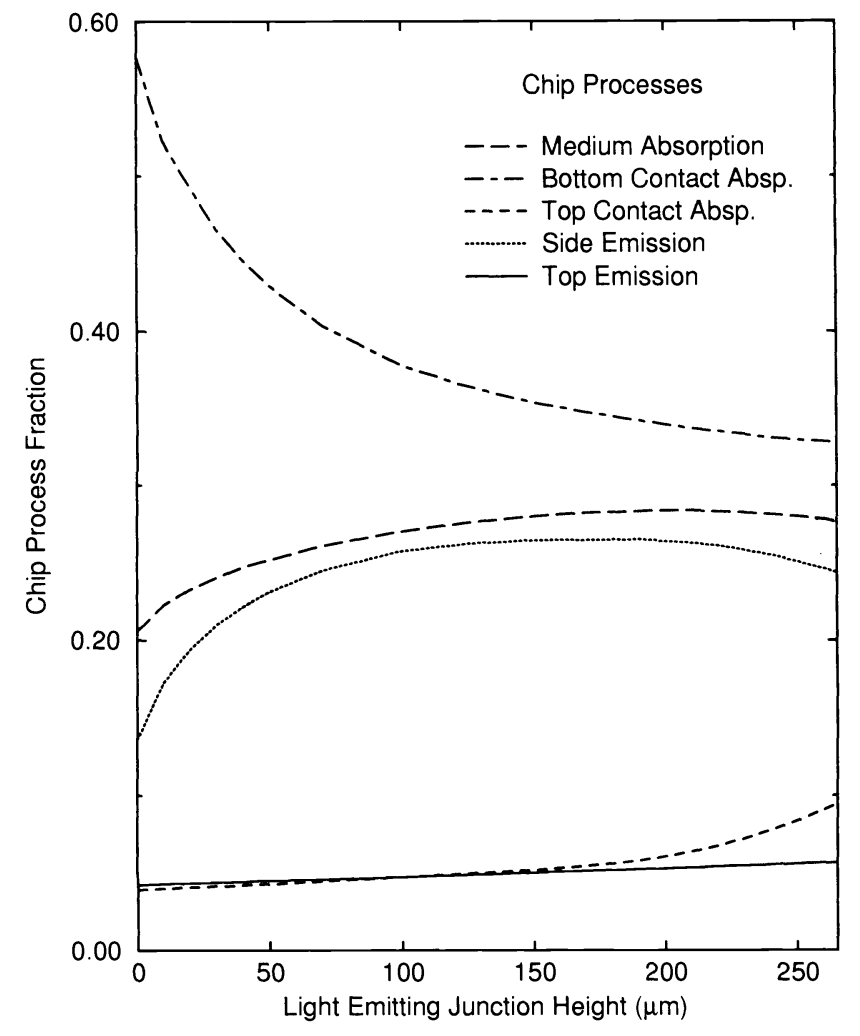

Fig. 4 Fractions of photons undergoing various processes in the semiconductor chip as functions of light-emitting junction height.

tion height for which bottom contact absorption is very high. Note that for all junction heights, side emission accounts for most of the transmitted photons.

We examine the specific cases of $h_{\text {junct }}=10 \mu \mathrm{m}$ and $h_{\text {junct }}=200 \mu \mathrm{m}$ in more detail. For the $h_{\text {junct }}=10 \mu \mathrm{m}$ structure, the fractions of photons transmitted through the four side surfaces and the top surface are 0.172 and 0.0429 , respectively; and for $h_{\text {junct }}=200 \mu \mathrm{m}, 0.264$ and 0.0527 . We might expect the emission intensities from a set of surfaces to be proportional to the surface areas, as would be the case if the photon flux inside the chip is heavily randomized by multiple internal reflections. For our geometry, the ratio between the total area of the four side surfaces and the exposed area of the top surface is 4.95 . The ratio between the side surfaces and the top surface emission intensities is 4.01 for the $h_{\text {junct }}=10 \mu \mathrm{m}$ structure and 5.01 for the $h_{\text {junct }}=200 \mu \mathrm{m}$ structure. This indicates that the photon flux inside the chip is not always well randomized. Indeed, this notion is con- firmed by statistics collected from our simulation. Table 2 shows averages and standard deviations for number of photon traversal segments (e.g., a three-segment process involves two internal reflections before absorption or transmission) for various chip processes. We see that for either structure, most of the emitted photons do not undergo many internal reflections; the same is also true for absorbed photons. Figure 5 shows the distribution of photons emitted from the top surface and one of the four side surfaces with respect to the number of photon traversal segments in the chip. The results for both the $h_{\text {junct }}=10 \mu \mathrm{m}$ and the $h_{\text {junct }}=200 \mu \mathrm{m}$ structures are shown for comparison. Almost all of the emitted photons escape in three traversal segments or less. Although most of these escape directly with just one traversal segment, a significant fraction does encounter one or two internal reflections before exiting the chip. The distribution patterns are noticeably different for the two structures, and the top and side surface distribution patterns also differ considerably. For all of these reasons, neither of the analytical models mentioned earlier is particularly suited for treating these cases.

Figure 5 shows that the number of direct (one-segment) side-exit photons for the $h_{\text {junct }}=10 \mu \mathrm{m}$ structure is approximately $2 / 3$ of that for the $h_{\text {junct }}=200 \mu \mathrm{m}$ structure. This is somewhat surprising as we might have intuitively expected this ratio to be closer to $1 / 2$, because half of the photons generated in a low-junction structure would be absorbed automatically by the substrate. Further simulations show that if we lowered the junction height to below $1 \mu \mathrm{m}$, the ratio does indeed approach $1 / 2$. However, the ratio increases rapidly as the junction is raised.

Figure 6 shows angular distributions of photons emitted from the top and side surfaces for these two structures. Note that the distribution at each $\theta$ includes contributions from all polar angles $\phi$, ranging from 0 to $2 \pi$, and therefore a normalizing factor of $1 / \sin \theta$ must be applied if we wish to look at distribution per unit solid angle. The top surface emission patterns for the two cases are similar, with the $h_{\text {junct }}=200 \mu \mathrm{m}$ case having somewhat higher intensity because of the proximity of the emission plane to the top surface. The side emission patterns are qualitatively different. Figure 7 shows the percentage of side-surface-emitted photons with $\theta>90 \mathrm{deg}$, as a function of light-emitting junction height. In the $h_{\text {junct }}=10 \mu \mathrm{m}$ case, $22 \%$ of the photons emitted by the side surfaces have $\theta>90 \mathrm{deg}$; in the $h_{\text {junct }}=200 \mu \mathrm{m}$ case, $59 \%$. In the $h_{\text {junct }}=10 \mu \mathrm{m}$ structure, the emission plane is fairly close to the absorbing bottom contact, and therefore only a small fraction of the downward pointing photons generated at the junction can escape directly; other photons must go through multiple internal reflections to be emitted at $\theta>90$

Table 2 Statistics on number of photon traversal segments for various processes in the semiconductor chip. Mean and standard deviation are displayed as $\mu \pm \sigma$.

\begin{tabular}{|l|c|c|r|r|r|}
\hline \hline$h_{\text {junct }}$ & Absorption & Absorption & Absorption & Emission & Emission \\
\hline $10 \mu \mathrm{m}$ & $2.87 \pm 2.65$ & $1.73 \pm 1.76$ & $2.45 \pm 1.54$ & $1.24 \pm 0.60$ & $1.55 \pm 0.62$ \\
\hline $200 \mu \mathrm{m}$ & $2.64 \pm 2.38$ & $2.94 \pm 1.81$ & $1.32 \pm 0.87$ & $1.33 \pm 0.69$ & $1.16 \pm 0.38$ \\
\hline \hline
\end{tabular}




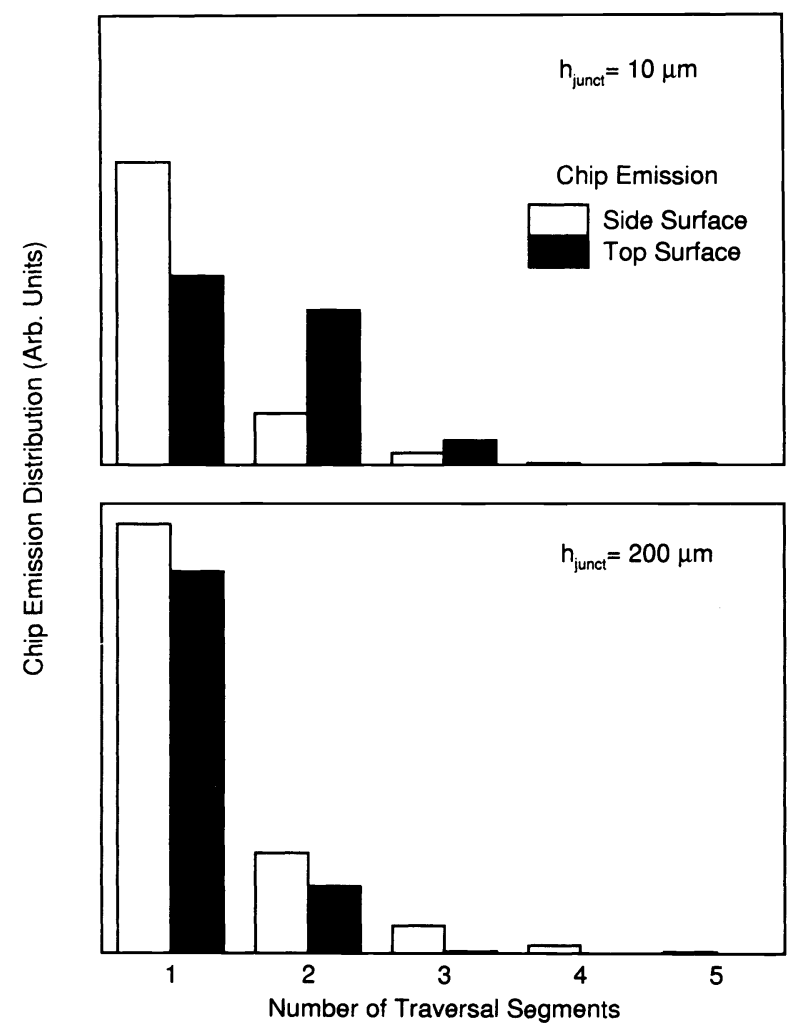

Fig. 5 Distribution of photons emitted from the LED top surface and a side surface with respect to the number of photon traversal segments in the chip. Results for two different light-emitting junction heights, $h_{\text {junct }}=10 \mu \mathrm{m}$ and $h_{\text {junct }}=200 \mu \mathrm{m}$, are shown.

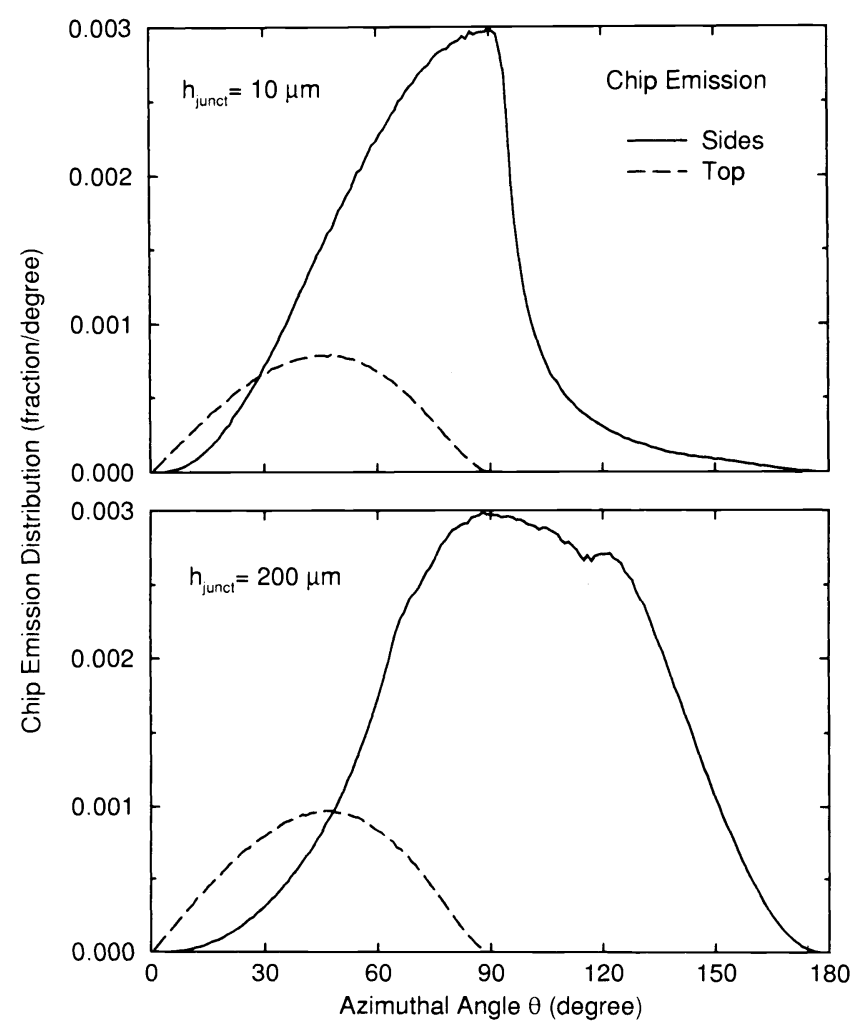

Fig. 6 Azimuthal distributions of photons emitted from the LED chip. The emission from the top surface is kept separately from the side surfaces. Results for two different light-emitting junction heights, $h_{\text {junct }}=10 \mu \mathrm{m}$ and $h_{\text {junct }}=200 \mu \mathrm{m}$, are shown.

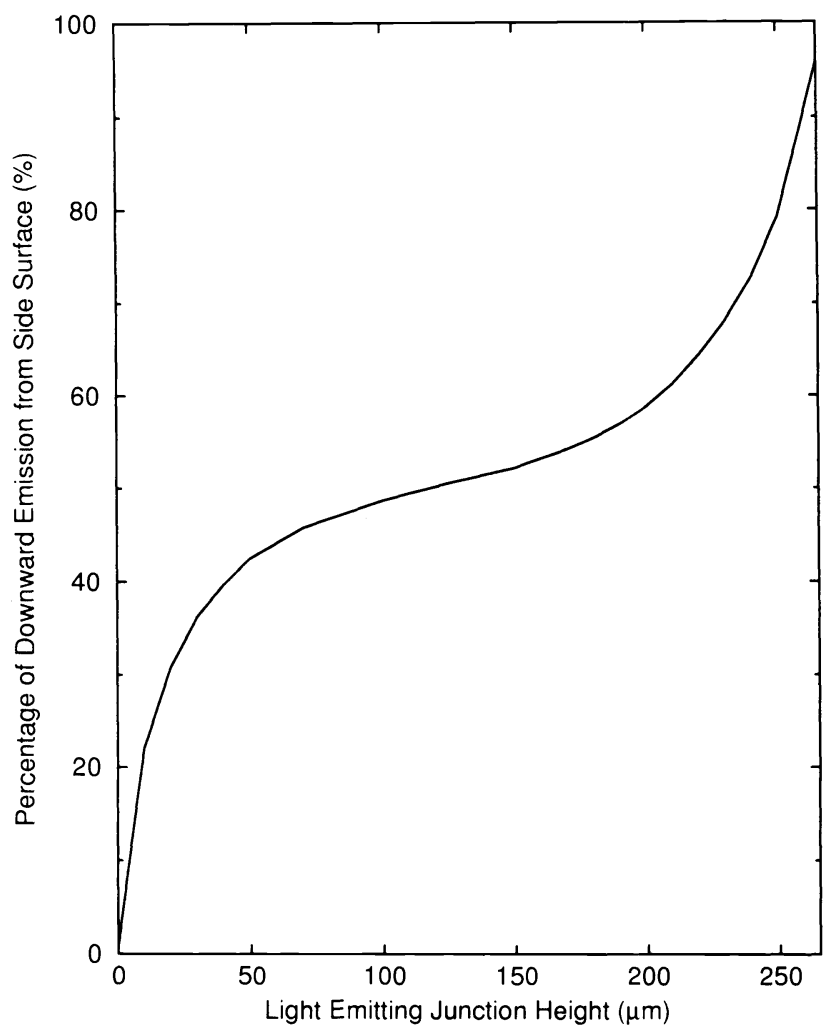

Fig. 7 Percentage of side surface emitted photons with $\theta>90$ deg, as function of light-emitting junction height.

deg. The side-surface-emission distribution for the $h_{\text {junct }}=200 \mu \mathrm{m}$ structure appears bimodal. In this structure, both upward- and downward-pointing photons generated at the junction can escape directly. In addition, because the junction is fairly close to the top surface, many photons can internally reflect off the top surface into the side surfaces and be transmitted at $\theta>90 \mathrm{deg}$. These two dominant mechanisms correspond to the one- and two-segment photons in Fig. 5, and they give rise to the bimodal appearance in the azimuthal distribution. The bimodal distribution leads us to conclude that simple analytical models assuming Lambertian sources may not adequately describe the surface emission patterns seen in our structures. Figure 8 shows polar plots of emission patterns from the top and side surfaces for our two structures. For comparison, the Lambertian emission patterns (circular) are also included. Although the top surface emission patterns are approximately Lambertian, the side surfaces emission patterns show noticeable deviations from Lambertian patterns.

Photons emitted from the chip may undergo one of the processes illustrated in Fig. 2. Figure 9 shows the fraction of photons (out of all photons generated) participating in the various processes as functions of light-emitting junction height. The dominant processes are direct escape from chip without striking the reflector dish and reflector dish sidewall (cone) reflection. Both of these processes follow similar initial increasing trends as the junction is raised from the chip bottom. However, the two curves diverge dramatically for $h_{\text {junct }}>180 \mu \mathrm{m}$; the cone-reflection fraction increases more rapidly, whereas the fraction for direct emission decreases. This can be explained with the aid of Fig. 7. As the light- 


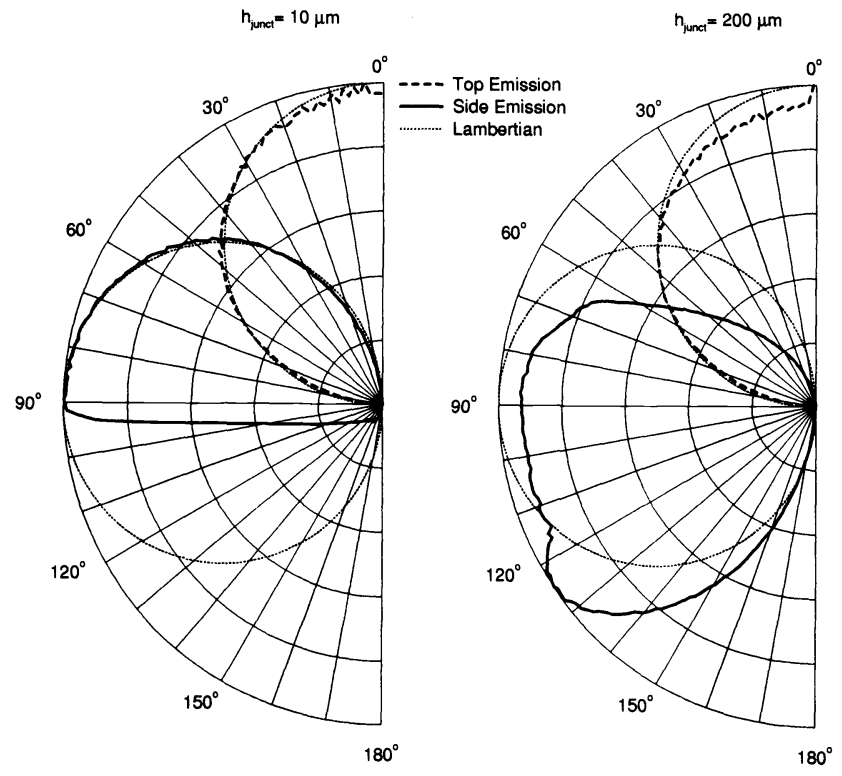

Fig. 8 Polar plot of relative intensity versus azimuthal angle for top and side surface emission from the LED chip. Top and side surface emission intensities are normalized separately. Results for two different light-emitting junction heights, $h_{\text {junct }}=10 \mu \mathrm{m}$ and $h_{\text {junct }}=200$ $\mu \mathrm{m}$, are shown.

emitting plane is moved closer to the chip top surface, side surfaces emit fewer upward pointing $(\theta<90 \mathrm{deg})$ photons that can escape directly, and more downward pointing $(\theta>$ $90 \mathrm{deg}$ ) photons that tend to reflect off the cone. For example, for the $h_{\text {junct }}=265 \mu \mathrm{m}$ structure, $96 \%$ of the photons emitting by the side surfaces have $\theta>90 \mathrm{deg}$. It is easy to understand why most of the photons emitted by the side surfaces have $\theta>90 \mathrm{deg}$ for large values of $h_{\text {junct }}$. As explained earlier, if the light-emitting junction is close to the top, then the side surface emission comes from two major contributions: those emitted directly from the junction and those internally reflecting off the top surface into the side surfaces at a downward angle; only the first mechanism can give rise to photons with $\theta<90 \mathrm{deg}$. However, any such photon must go through the portion of the side surface above the emission plane. But this portion is diminished as the emission plane approaches the top, leading to fewer and fewer side surface emitted photons with $\theta<90 \mathrm{deg}$.

Figure 10 shows the angular distribution of photons emitted from the chip-dish assembly for the specific cases of $h_{\text {junct }}=10 \mu \mathrm{m}$ and $h_{\text {junct }}=200 \mu \mathrm{m}$. Because the reflector dish is designed to direct photons toward the forward direction, all photons have $\theta<90 \mathrm{deg}$. Again, we see that the direct escape and cone reflection processes dominate. The emission characteristics for the two junction height are quite different. In particular, the cone reflection spectra for the $h_{\text {junct }}=10 \mu \mathrm{m}$ and $h_{\text {junct }}=200 \mu \mathrm{m}$ structures peak at 28 and $14 \mathrm{deg}$, respectively.

Figure 11 shows the fractions of photons exiting through different surfaces of the epoxy lens as functions of junction height. Note that although emission through the hemispherical surface dominates, a significant number of photons do exit through the bottom, and are presumably rendered useless. For instance, for the $h_{\text {junct }}=190 \mu \mathrm{m}$ structure, over $14 \%$ of the emitted photons are lost through the bottom. For appli-

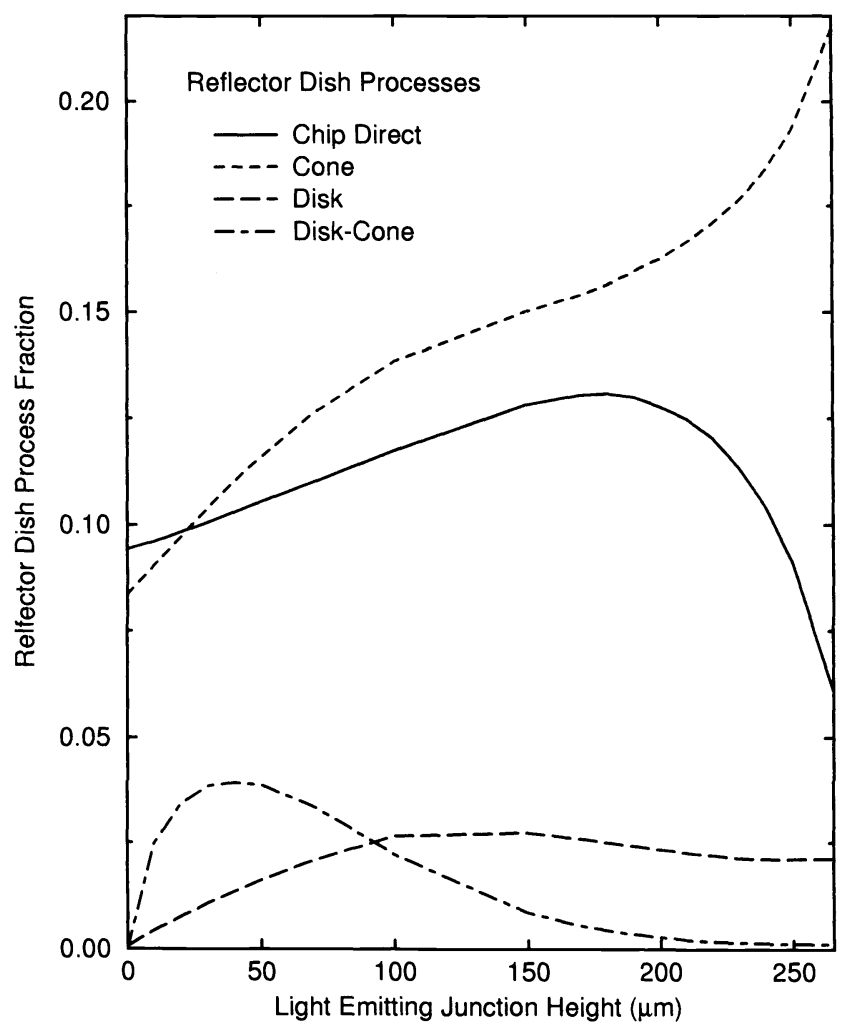

Fig. 9 Fractions of photons exiting the reflector dish via modes are shown in Fig. 2, as functions of light-emitting junction height.

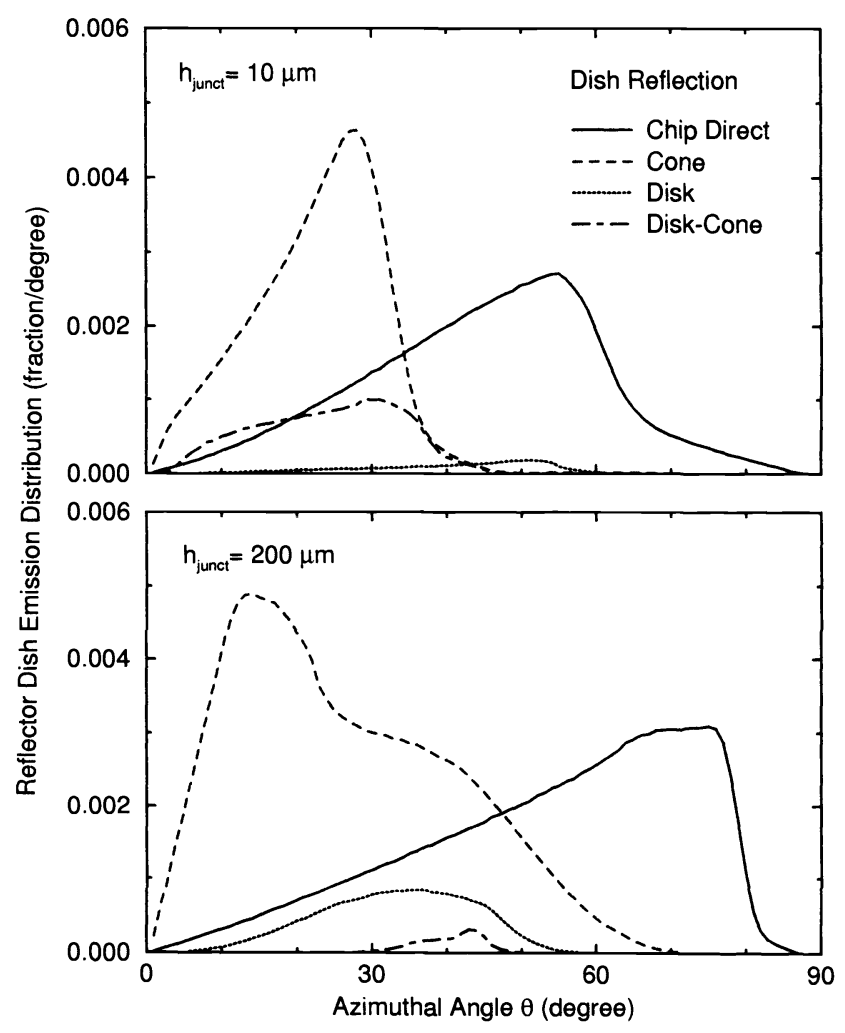

Fig. 10 Azimuthal distributions of photons emitted from the LED chip/reflector dish assembly for the four exit modes shown in Fig. 2. 


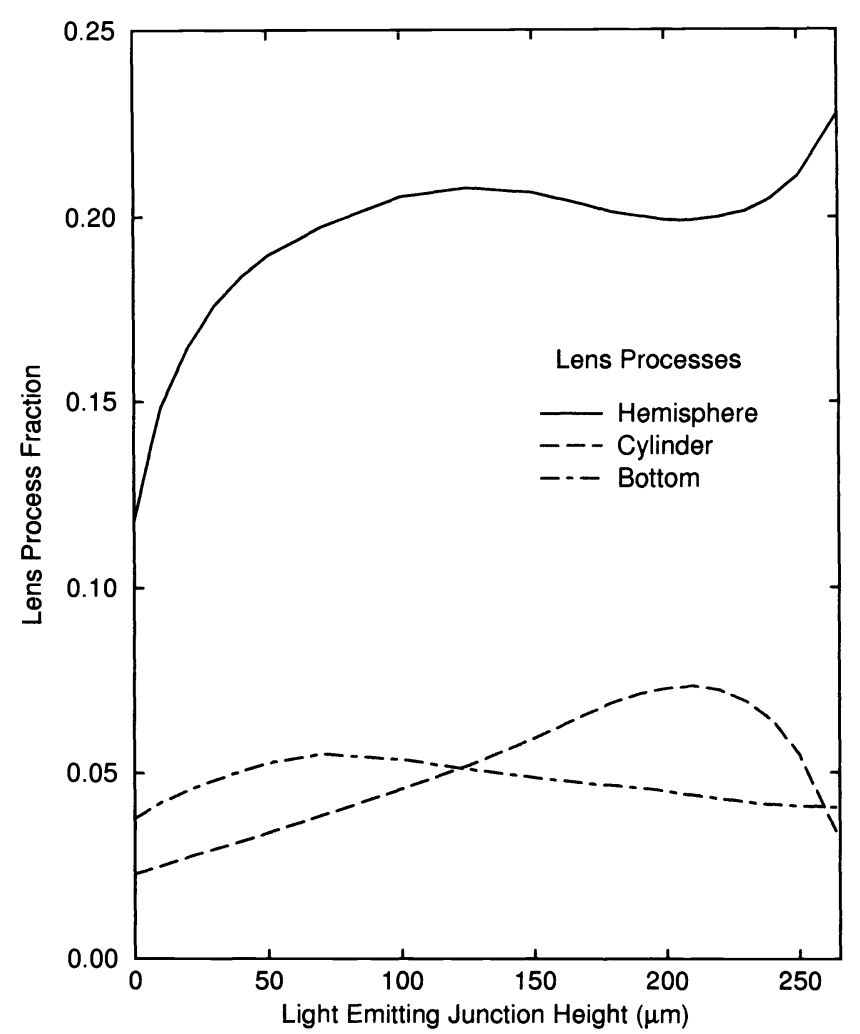

Fig. 11 Fractions of photons exiting the epoxy lens via the top hemisphere, the cylinder wall, and the bottom disk; plotted as functions of light-emitting junction height.

cations, it is important to examine the emission patterns from the lens. Figure 12 shows the azimuthal distributions of photons emitted through the top hemisphere and the cylinder wall for the $h_{\text {junct }}=10 \mu \mathrm{m}$ and $h_{\text {junct }}=200 \mu \mathrm{m}$ structures. Only the forward directions $(\theta<90 \mathrm{deg})$ are shown. Note that most of the photons emitted into the forward directions are transmitted through the top hemisphere. The hemispherical lens also focuses light into $\theta<20 \mathrm{deg}$.

Simulation statistics show that most of the photons transmitted through the hemisphere either exit directly or after a single internal reflection off the cylinder wall, whereas most of the photons transmitted through the cylinder wall exit directly. Photons exiting through the bottom typically go through a higher number of internal reflections. Table 3 shows averages and standard deviations for number of photon traversal segments in the epoxy for the three lens exit modes.

Figure 13 summarizes the emission patterns for the $h_{\text {junct }}=10 \mu \mathrm{m}$ and $h_{\text {junct }}=200 \mu \mathrm{m}$ structures by showing the total emission after each of the three simulation stages (chip, dish, and lens). Because there is essentially no loss after photons are emitted from the chip, the areas under the three distribution curves are the same, all yielding the value of the extraction efficiency. However, the shapes of the curves are quite distinct. The chip emission tends to be broadly distributed, the reflector dish brings all emission to $\theta<90 \mathrm{deg}$, and the lens tightly focuses a portion of the emission into $\theta<20$ deg, while scattering the remainder to a much broader distribution. For the $h_{\text {junct }}=10 \mu \mathrm{m}$ structure, $56 \%$ of the photons are emitted with $\theta<20 \mathrm{deg}$, and $75 \%$ of the photons are emitted with $\theta<90 \mathrm{deg}$; for the $h_{\text {junct }}=200 \mu \mathrm{m}$ structure,

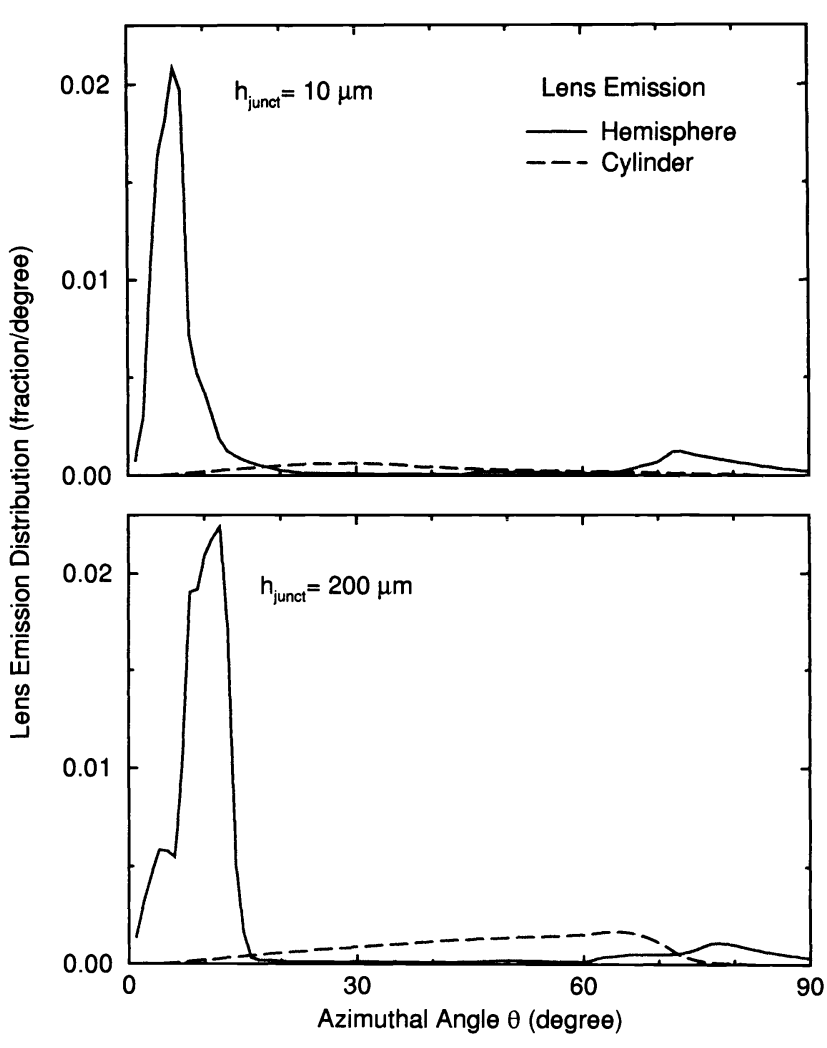

Fig. 12 Azimuthal distributions of photons emitted from the epoxy lens through the top hemisphere and the cylinder wall.

corresponding values are 54 and $82 \%$, respectively. These percentages can be adjusted by changing the epoxy index of refraction. Lowering the index would decrease internal reflection in the lens, and thus increase forward direction emission. However, this would also increase the chip-epoxy index mismatch, resulting in decreased photon emission from the chip. Of course, how to make an effective trade-off in situations such as this is precisely the type of question that we hope to be able to answer with our simulations.

Before concluding our discussion, we briefly examine the effect of current spreading resistance. It has been demonstrated that when the light-emitting junction is close to the top surface, the emission from the junction is nonuniform because of limited current spreading. ${ }^{8}$ By producing proportionally more photons underneath the absorbing top contact, this effect can reduce light extraction efficiency. Nonuniform emission from the light-emitting junction plane can be included in our model for more realism.

We conclude by pointing out some areas where we could refine our model to make it more realistic and versatile. These include (1) generalized lens geometry, (2) heterostructure chips, (3) polychromatic emission spectra from lightemitting junctions, (4) nonuniform photon generation on light-emitting plane, (5) roughness on chip surfaces, (6) partially reflecting contacts, (7) photon recycling effects, and (8) photon re-entrance to semiconductor chip. Most of these improvements can be made in a straightforward manner, and will be implemented in the near future. We briefly discuss some of these topics here. Chip surface roughness effects must be included in realistic simulations. In some chips, horizontal saw marks on the chip side surfaces are clearly visible 
Table 3 Statistics on number of photon traversal segments in epoxy lens for various exit modes. Mean and standard deviation are displayed as $\mu \pm \sigma$.

\begin{tabular}{|c|c|c|c|}
\hline \hline$h_{\text {junct }}$ & Hemisphere & Cylinder & Bottom \\
\hline $10 \mu \mathrm{m}$ & $1.50 \pm 1.43$ & $1.09 \pm 0.48$ & $3.97 \pm 1.58$ \\
\hline $200 \mu \mathrm{m}$ & $1.43 \pm 1.36$ & $1.07 \pm 0.38$ & $4.17 \pm 1.57$ \\
\hline \hline
\end{tabular}

under the optical microscope. We expect that by including these roughness patterns, the emission characteristics would become more diffuse, but less isotropic. The implementation of partially reflecting contacts should also be important for our model. Figure 4 shows that by assuming contacts are totally absorbing, more than half of the photons generated are lost to contact absorption. The inclusion of partially reflecting contacts could noticeably increase the number of transmitted photons. Electron-hole pairs generated by medium absorption may recombine to produce more photons; this photon recycling effect might be important in some material systems with high internal quantum efficiency, and should be included in the model. Another important issue is of photon re-entrance; later we discuss some results from a preliminary study on the topic. For simplicity and speed, our simulation currently uses a sequential model, which assumes that once a photon leaves the semiconductor chip, the chip can be ignored in the ray-tracing process; the reflector dish is treated the same way. However, it is certainly possible that a photon could re-enter a chip after it is emitted. Figure 14

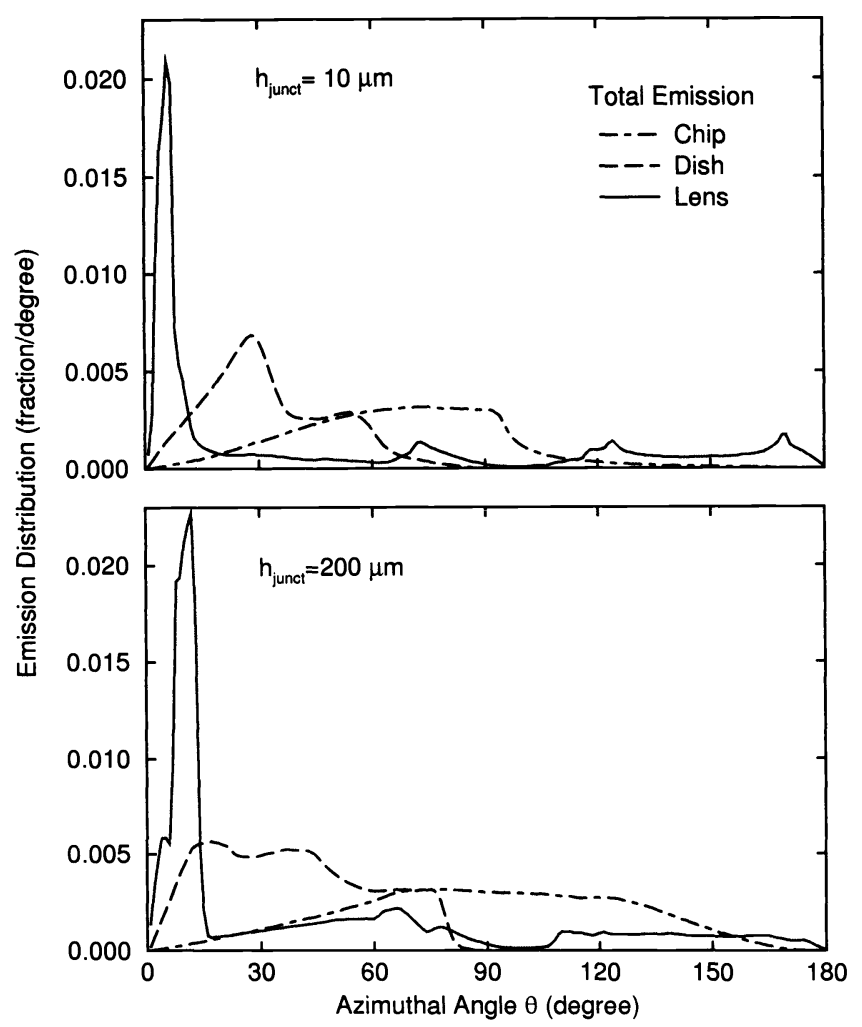

Fig. 13 Azimuthal distributions of all photons emitted from the LED chip, the reflector dish, and the epoxy lens. shows the fraction of photons reflected from the dish back to the LED chip, as a function of light-emitting junction height. Note that for junction heights below $50 \mu \mathrm{m}$, less than $1 \%$ of the photons are reflected back to the chip. In that case, the sequential model is quite valid. However, the dish-tochip fraction increases to over $10 \%$ for junction height over $220 \mu \mathrm{m}$, and there the validity of the sequential model is less certain. Many of the dish-to-chip photons would re-enter the chip, and quite possibly be absorbed inside the chip, thus lowering the extraction efficiency. Issues such as these remain to be studied. Overall, we are optimistic that once these issues are resolved, our method could be used as a computer-aided design tool for optimizing light-extraction efficiencies in LEDs, and for designing LED spatial emission characteristics.

\section{Summary}

In this paper we employ a Monte Carlo ray-tracing technique to model light-extraction characteristics of light-emitting

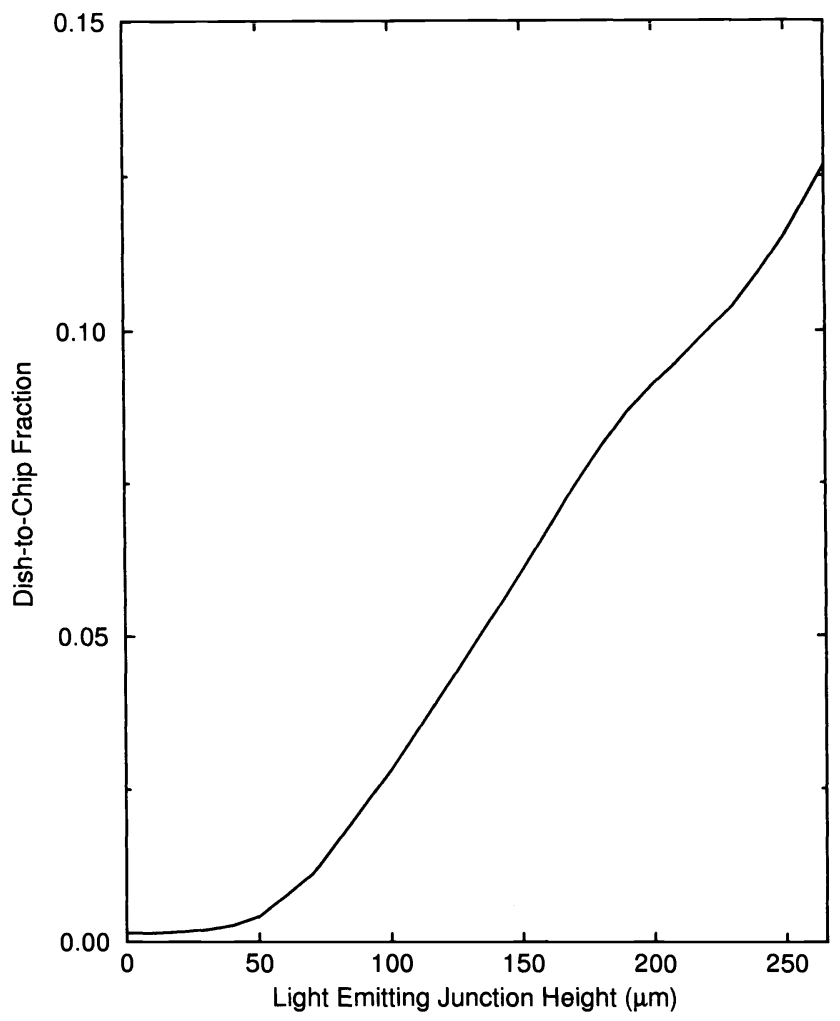

Fig. 14 Fraction of photons reflected from the dish back to the LED chip, as a function of light-emitting junction height. 
diodes. By effectively utilizing readily available computational resources and relaxing restrictive assumptions on photon traversal history, our method improves on available analytical models for estimating light-extraction efficiencies from bare LED chips and enhances modeling capabilities by realistically treating the various processes that photons can encounter in a packaged LED. Our method is not only capable of calculating extraction efficiencies, but can also provide extensive statistical information on photon extraction processes and predict LED spatial emission characteristics. Simulations using our method can be performed very rapidly on modern workstations, making it a good candidate for an effective design tool. As an example, we performed a series of simulations to optimize light-extraction efficiency with respect to light-emitting junction height in a simple LED structure. We showed that by carefully analyzing the extensive statistics collected during the simulation, we are able to single out the processes that are important in determining lightextraction efficiency and identify the mechanisms that give rise to the particular spatial emission characteristics in each structure. We also pointed out several areas where our model could be improved to make it more realistic and versatile. We hope to be able to implement these improvements in the near future, and validate the model by comparison with experimental observations. We believe that, with some refinement, our method could become a valuable LED design tool.

\section{Acknowledgments}

The authors would like to thank J. F. Swenberg, M. W. Wang, F. M. Steranka, M. G. Craford, and P. Mahowald for helpful discussions. This work was supported by the Office of Naval Research (ONR) under Grant No. N00014-92-J-1845.

\section{References}

1. K. Werner, "Higher visibility of LEDs," IEEE Spectrum, 30-39 (July 1994).

2. M. G. Craford, "LEDs challenge the incandescents," IEEE Circ. Devices 8, 24-29 (September 1992).

3. W. N. Carr, "Photometric figures of merit for semiconductor luminescent sources operating in spontaneous decay mode," Infrared Phys. 6, 1-19 (1966).

4. W. B. Joyce, R. Z. Bachrach, R. W. Dixon, and D. A. Sealer, "Geometrical properties of random particles and the extraction of photons from electroluminescent diodes," J. Appl. Phys. 45(5), 2229-2253 (1974).
5. S. Gage et al., Optoelectronics/Fiber-Optics Applications Manual, McGraw-Hill, New York (1981).

6. D. Badouel, "An efficient ray-polygon intersection," in Graphics Gems, A.S. Glassner, Ed., pp. 390-399, Academic Press, Boston (1990).

7. E. Hecht, Optics, 2nd ed., Addison-Wesley, Reading, MA (1987).

8. R. M. Fletcher, C. P. Kuo, T. D. Osentowski, K. H. Huang, M. G. Craford, and V. M. Robbins, "The growth and properties of high performance AlGaInP emitters using a lattice matched $\mathrm{GaP}$ window layer," J. Elect. Mater. 20, 1125-1130 (1991).

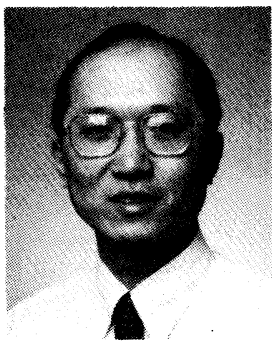

David Z.-Y. Ting received a BS degree (with honors) in physics from the California Institute of Technology (Caltech), Pasedena, in 1980 and MS and PhD degrees in physics from the University of Illinois at Urbana-Champaign in 1981 and 1986, respectively. He is currently a senior research fellow in the Department of Applied Physics at Caltech. His research activities include the theoretical studies of electronic and optical properties of semiconductor alloys, quantum wells, and superlattices; modeling of heterostructure tunnel devices; quantum transport in nanostructure; and optical simulations. Results of his research have been reported in over 50 research publications and in many technical presentations. Dr. Ting is a member of Tau Beta Pi, Phi Kappa Phi, Sigma Xi, and the American Physical Society.

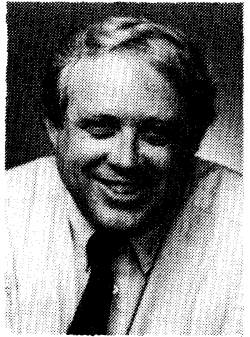

Thomas C. McGill is currently the Fletcher Jones Professor of Applied Physics at the California Institute of Technology (Caltech). Before joining the faculty at Caltech in 1971, he held an AFNRC postdoctoral fellowship at Princeton University and a North Atlantic Treaty Organization postdoctoral fellowship at the University of Bristol. He obtained his MS and PhD degrees in electrical engineering from Caltech in 1965 and 1969, respectively, and a BS in mathematics in 1963 and a BS in electrical engineering in 1964 , both from Lamar State College of Science. Professor McGill's current research interests are in the broad area of microstructures for modern electronics. His earlier research included many areas of semiconductor physics and devices. Results of his research have been reported in over 300 research publications and in numerous technical presentations. Professor McGill was a member of the OTS/ APS Committee on Solar Photovoltaics and the Solid State Panel. $\mathrm{He}$ is a member of the Advanced Research Project Agency's Defense Science Research Council, and is the former chairman of its Steering Committee. He is a member of the Naval Studies Board Committee on Electronics Research. He is a fellow of the APS and a member of IEEE, AAAS, and Sigma Xi. 